\section{Bioprospecting for drugs}

Sir - In an article on bioprospecting ${ }^{1}$, one of us (D. N.) was quoted to the effect that "only one sample in 250,000 will directly yield a commercial drug, although many more samples may serve as useful leads for modification through combinatorial chemistry". It was not made clear that this estimate is based on data from the antibiotics industry $\mathrm{y}^{2,3}$ and refers specifically to drug discovery from microbial sources. These data should not be translated directly to other natural sources, such as plants and marine organisms.

With estimates of the number of terrestrial plant species ranging from 250,000 to 400,000 , the implication would be that only one or two commercial anticancer drugs might be developed from this source. This is an erroneous conclusion.

Indeed, the experience of the US National Cancer Institute (NCI) with the screening of plants between 1960 and 1982 indicates that some 35,000 samples (leaves, bark, roots and so on), derived from an estimated 12,000 species collected mainly from temperate regions, yielded two highly significant anti-cancer drugs, paclitaxel (Taxol) and camptothecin (which has been converted so far into two commercial products, topotecan and irinotecan), and homoharringtonine, which shows significant clinical efficacy against several leukaemias. This gives a success rate of one in about 4,000 species tested for activity in one disease category. A substantial improvement in the 'hit' rate can be expected with expanded screening.

We wish to emphasize that the NCI is continuing to explore the potential of nature as a source of novel pharmacophores in collaboration with the extramural scientific community. Where feasible, collection contracts are being replaced by direct collaboration with qualified organizations in the source countries, particularly in the investigation of plants. Gordon M. Cragg

David J. Newman

Stringner Sue Yang

Natural Products Branch,

Division of Cancer Treatment and Diagnosis,

National Cancer Institute,

PO Box B,

Frederick,

Maryland 21702-1201, USA

e-mail:cragg@dtpax2.ncifcrf.gov

1. Macilwain, C. Nature 392, 535-540 (1998).

2. Omura, S. in The Future of Antibiotherapy and Antibiotics Research (eds Bost, P. E. et al.) 389-405 (Academic, New York, 1981).

3. Rake, J. B. et al. J. Antibiotics 39, 58-67 (1986).

\title{
No convergence for references
}

Sir - Here are a few examples of how the reference to a hypothetical paper entitled "Europe and Euro" would appear in different scientific journals:

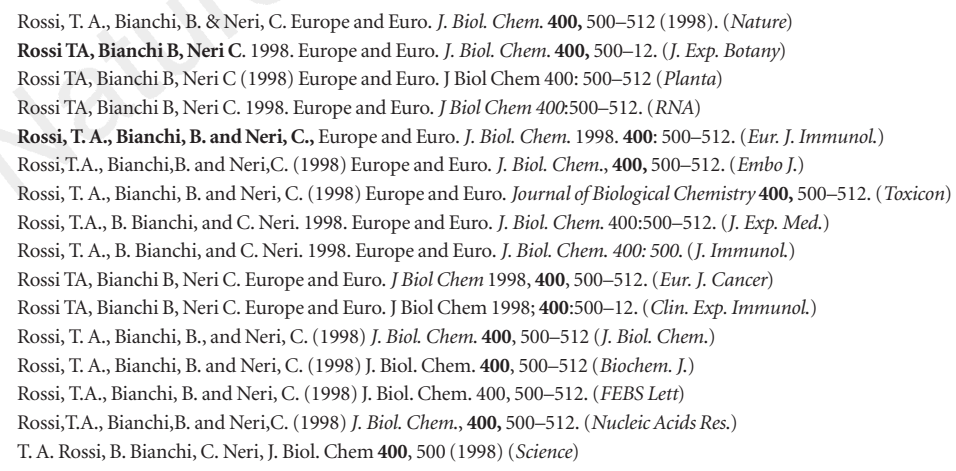

The list could be longer. And we have not taken into account the two alternatives for bibliography: alphabetical list of references or numbers in order of appearance. In the latter case the numbers may be 1 or 1 . or [1], etc.

We propose that the year of Euro, in which 11 countries (and we hope they will soon be more) join in the use of the same currency, should also be the year in which scientific journals in Europe (and possibly throughout the world) join in the same way of handling bibliography.

\section{Simonetta Sperti}

\section{Fiorenzo Stirpe}

\section{Maurizio Brigotti}

Dipartimento di Patologia sperimentale,

Università di Bologna, Bologna, Italy

e-mail:stirpef@alma.unibo.it

\section{No laughing matter}

Sir - Andrew Birch's pun (Nature 392, 745; 1998) on gas and bubble chambers trivializes genocide in much the same spirit as the revisionists who are the subject of the accompanying article. "Revisionism" appears there euphemistically for the support of Nazi political aims and their contemporary offshoots. Birch's belief that gas chambers are now worth a thighslapper indicates irresponsible ignorance or disregard of recent history.

I hope something will be done to prevent further cartoons in Nature joking about the other methods of the Holocaust to degrade and destroy its victims, for example beating, shooting, starvation and so on. Reflection by some on the historical role of science, especially in this century, has led to attempts to humanize the relation between science and society. Birch's cartoon does not add to the discussion.

\section{Michael G. Vicker}

Biology/Chemistry,

University of Bremen,

D-28359 Bremen,

Germany

e-mail:vicker@uni-bremen.de

Andrew Birch replies: Far from trivializing genocide, the object of my cartoon was to ridicule the revisionists and the French National Front, and I feel that in that context the pun was justified. Just because a subject is of the utmost seriousness surely does not mean that one cannot use humour to make a political point. I obviously regret giving offence to Michael Vicker, but I hope the revisionists were more offended.

Andrew Birch

Calle Gigantes 19,

29008 Malaga,

Spain

\section{Unreliable errors}

Sir - The article on Emil Abderhalden and his fraudulent "defence enzymes" (Nature 393, 109-111; 1998) brought back memories. When I was a graduate student at Columbia University in 1950, engaged in developing methods of peptide synthesis, I found that almost invariably I could not repeat Abderhalden's experiments.

In one of my most frustrated moods, I asked my mentor, Dr Erwin Brand, if Abderhalden was always wrong. "No," he said. "He is not that reliable".

Bernard F. Erlanger

Department of Microbiology,

Columbia University,

701 West 168 Street,

New York, New York 10032, USA 